OPEN ACCESS

Approved by:

Frontiers Editorial Office,

Frontiers Media SA, Switzerland

${ }^{*}$ Correspondence: Keietsu Abe

keietsu.abe.b5@tohoku.ac.jp

Specialty section:

This article was submitted to Fungal Physiology and Metabolism,

a section of the journal

Frontiers in Fungal Biology

Received: 10 February 2022 Accepted: 11 February 2022 Published: 07 March 2022

Citation:

Miyazawa K, Yamashita T, Takeuchi A

Kamachi Y, Yoshimi A, Tashiro Y,

Koizumi A, Ogata M, Yano $S$,

Kasahara S, Sano M, Yamagata Y,

Nakajima T and Abe K (2022)

Corrigendum: $A$

Glycosylphosphatidylinositol-

Anchored $\alpha$-Amylase Encoded by amy $D$ Contributes to a Decrease in

the Molecular Mass of Cell Wall $\alpha-1,3-G / u c a n$ in Aspergillus nidulans.

Front. Fungal Biol. 3:872790.

doi: 10.3389/ffunb.2022.872790

\section{Corrigendum: A} \section{Glycosylphosphatidylinositol-} Anchored $\alpha$-Amylase Encoded by amyD Contributes to a Decrease in the Molecular Mass of Cell Wall $\alpha-1,3-$ Glucan in Aspergillus nidulans

\author{
Ken Miyazawa ${ }^{1,2}$, Takaaki Yamashita ${ }^{1}$, Ayumu Takeuchi ${ }^{1}$, Yuka Kamachi ${ }^{1}$, Akira Yoshimi ${ }^{3,4}$, \\ Yuto Tashiro ${ }^{1}$, Ami Koizumi ${ }^{1}$, Makoto Ogata ${ }^{5}$, Shigekazu Yano ${ }^{6}$, Shin Kasahara ${ }^{7}$, \\ Motoaki Sano ${ }^{8}$, Youhei Yamagata ${ }^{9}$, Tasuku Nakajima ${ }^{4}$ and Keietsu Abe ${ }^{1,4,10 *}$ \\ ${ }^{1}$ Laboratory of Applied Microbiology, Department of Microbial Biotechnology, Graduate School of Agricultural Science, \\ Tohoku University, Sendai, Japan, ${ }^{2}$ Laboratory of Filamentous Mycoses, Department of Fungal Infection, National Institute of \\ Infectious Diseases, Tokyo, Japan, ${ }^{3}$ Laboratory of Environmental Interface Technology of Filamentous Fungi, Graduate \\ School of Agriculture, Kyoto University, Kyoto, Japan, ${ }^{4}$ ABE-Project, New Industry Creation Hatchery Center, Tohoku \\ University, Sendai, Japan, ${ }^{5}$ Faculty of Food and Agricultural Sciences, Fukushima University, Fukushima, Japan, \\ ${ }^{6}$ Department of Biochemical Engineering, Graduate School of Engineering, Yamagata University, Yonezawa, Japan, ${ }^{7}$ Food \\ Microbiology Unit, School of Food and Agricultural Sciences, Miyagi University, Sendai, Japan, ${ }^{8}$ Genome Biotechnology \\ Laboratory, Kanazawa Institute of Technology, Hakusan, Japan, ${ }^{9}$ Department of Applied Life Science, The United Graduate \\ School of Agricultural Science, Tokyo University of Agriculture and Technology, Fuchu, Japan, ${ }^{10}$ Department of Microbial \\ Resources, Graduate School of Agricultural Science, Tohoku University, Sendai, Japan
}

Keywords: cell wall, filamentous fungi, Aspergillus nidulans, glycosylphosphatidylinositol-anchored protein, $\alpha$-amylase, $\alpha$-1, 3-glucan

\section{A Corrigendum on}

A Glycosylphosphatidylinositol-Anchored $\alpha$-Amylase Encoded by amyD Contributes to a Decrease in the Molecular Mass of Cell Wall $\alpha-1,3-$ Glucan in Aspergillus nidulans by Miyazawa, K., Yamashita, T., Takeuchi, A., Kamachi, Y., Yoshimi, A., Tashiro, Y., Koizumi, A., Ogata, M., Yano, S., Kasahara, S., Sano, M., Yamagata, Y., Nakajima, T., and Abe, K. (2022) Front. Fungal Biol. 2:821946. doi: 10.3389/ffunb.2021.821946

In the original article, "the funder the Japan Society for Promotion of Science (JSPS) KAKENHI, $20 \mathrm{H} 02895$ " to Keietsu Abe was not included. The corrected Funding statement appears below.

Funding

This work was supported by the Japan Society for Promotion of Science (JSPS) KAKENHI Grant Nos. 26292037 (KA), 18K05384 (KA), 20H02895 (KA) and 20K22773 (KM), and a Grant-in-Aid for JSPS Fellows Grant No. 18J11870 (KM). This work was also supported by the Institute for Fermentation, Osaka (Grant No. L-2018-2-014) (KA) and by the project JPNP20011 (KA), which is commissioned by the New Energy and Industrial Technology Development Organization (NEDO). 
The authors apologize for this error and state that this does not change the scientific conclusions of the article in any way. The original article has been updated.

Publisher's Note: All claims expressed in this article are solely those of the authors and do not necessarily represent those of their affiliated organizations, or those of the publisher, the editors and the reviewers. Any product that may be evaluated in this article, or claim that may be made by its manufacturer, is not guaranteed or endorsed by the publisher.

Copyright ( 2022 Miyazawa, Yamashita, Takeuchi, Kamachi, Yoshimi, Tashiro, Koizumi, Ogata, Yano, Kasahara, Sano, Yamagata, Nakajima and Abe. This is an open-access article distributed under the terms of the Creative Commons Attribution License (CC BY). The use, distribution or reproduction in other forums is permitted, provided the original author(s) and the copyright owner(s) are credited and that the original publication in this journal is cited, in accordance with accepted academic practice. No use, distribution or reproduction is permitted which does not comply with these terms. 